\title{
Evaluation of computed tomography in patients with atypical angina or chest pain clinically referred for invasive coronary angiography: randomised controlled trial
}

\begin{abstract}
Marc Dewey, ${ }^{1}$ Matthias Rief, ${ }^{1}$ Peter Martus, ${ }^{2}$ Benjamin Kendziora,, Sarah Feger, ${ }^{1}$ Henryk Dreger, ${ }^{1}$ Sascha Priem, ${ }^{1}$ Fabian Knebel, ${ }^{1}$ Marko Böhm, ${ }^{1}$ Peter Schlattmann, ${ }^{3}$ Bernd Hamm, ${ }^{1}$ Eva Schönenberger, Michael Laule, ${ }^{1}$ Elke Zimmermann¹
\end{abstract}

\section{ABSTRACT}

OBJECTIVE

To evaluate whether invasive coronary angiography or computed tomography (CT) should be performed in patients clinically referred for coronary angiography with an intermediate probability of coronary artery disease.

DESIGN

Prospective randomised single centre trial.

SETTING

University hospital in Germany.

\section{PARTICIPANTS}

340 patients with suspected coronary artery disease and a clinical indication for coronary angiography on the basis of atypical angina or chest pain.

\section{INTERVENTIONS}

168 patients were randomised to $\mathrm{CT}$ and 172 to coronary angiography. After randomisation one patient declined CT and 10 patients declined coronary angiography, leaving 167 patients (88 women) and 162 patients (78 women) for analysis. Allocation could not be blinded, but blinded independent investigators assessed outcomes.

\section{MAIN OUTCOME MEASURE}

The primary outcome measure was major procedural complications within 48 hours of the last procedure related to $\mathrm{CT}$ or angiography.

RESULTS

Cardiac CT reduced the need for coronary angiography from $100 \%$ to $14 \%$ (95\% confidence interval $9 \%$ to $20 \%, \mathrm{P}<0.001)$ and was associated with a significantly

\section{WHAT IS ALREADY KNOWN ON THIS TOPIC}

Computed tomography (CT) is an accurate non-invasive alternative to invasive coronary angiography

The risks and advantages of $\mathrm{CT}$ in patients with suspected coronary artery disease owing to atypical chest pain and a clinical indication for coronary angiography are unknown

\section{WHAT THIS STUDY ADDS}

In patients with atypical angina or chest pain suggestive of coronary artery disease, CT reduced minor but not major procedural complications compared with direct coronary angiography

The length of hospital stay was shortened by 22.9 hours using CT instead of coronary angiography whereas exposure to radiation was similar

$\mathrm{CT}$ resulted in a significantly increased diagnostic yield of coronary angiography, defined as the rate of obstructive coronary artery disease (75\% v15\%), and was a safe gatekeeper for coronary angiography with no increase in long term events greater diagnostic yield from coronary angiography: $75 \%$ (53\% to $90 \%$ ) v $15 \%$ (10\% to $22 \%$ ), $\mathrm{P}<0.001$. Major procedural complications were uncommon (0.3\%) and similar across groups. Minor procedural complications were less common in the CT group than in the coronary angiography group: $3.6 \%$ (1\% to $8 \%$ ) v $10.5 \%$ (6\% to $16 \%), P=0.014$. CT shortened the median length of stay in the angiography group from 52.9 hours (interquartile range 49.5-76.4 hours) to 30.0 hours (3.5-77.3 hours, $\mathrm{P}<0.001)$. Overall median exposure to radiation was similar between the $\mathrm{CT}$ and angiography groups: $5.0 \mathrm{mSv}$ (interquartile range 4.2-8.7 mSv) $v 6.4 \mathrm{mSv}$ (3.4-10.7 mSv), $P=0.45$. After a median follow-up of 3.3 years, major adverse cardiovascular events had occurred in seven of 167 patients in the CT group (4.2\%) and six of $162(3.7 \%)$ in the coronary angiography group (adjusted hazard ratio 0.90, 95\% confidence interval 0.30 to $2.69, \mathrm{P}=0.86$ ). $79 \%$ of patients stated that they would prefer CT for subsequent testing. The study was conducted at a University hospital in Germany and thus the performance of CT may be different in routine clinical practice. The prevalence was lower than expected, resulting in an underpowered study for the predefined primary outcome.

\section{CONCLUSIONS}

CT increased the diagnostic yield and was a safe gatekeeper for coronary angiography with no increase in long term events. The length of stay was shortened by 22.9 hours with CT, and patients preferred noninvasive testing.

TRIAL REGISTRATION

ClinicalTrials.gov NCT00844220.

\section{Introduction}

The final diagnosis of obstructive coronary artery disease is made by invasive coronary angiography. ${ }^{1}$ Coronary angiography has the advantages of allowing simultaneous coronary stenting and planning of coronary artery bypass grafting. Evidence from the United States $^{2}$ and Europe, ${ }^{3}$ however, suggests that invasive coronary angiography has low diagnostic yield, indicating potential overuse, and also rare but possibly life threatening complications. ${ }^{45}$ Computed tomography (CT) is the most accurate non-invasive test for the diagnosis of coronary artery disease. ${ }^{6}$ The greatest clinical value of cardiac CT may be its ability to reliably rule out obstructive coronary artery disease in patients with atypical presentation and thus with a low-to-intermediate pretest probability of disease. ${ }^{78}$ 
In a large multicentre US trial, CT did not improve clinical outcomes compared with functional testing, ${ }^{9}$ whereas in the large SCOT-Heart (Scottish COmputed Tomography of the HEART) trial in rapid access chest pain clinics, ${ }^{10}$ CT used for diagnosis and management decisions was found to possibly reduce myocardial infarction on follow-up of patients with recent onset angina. ${ }^{11}$ CT may also lead to fewer angina symptoms, downstream testing, and costs, while increasing exposure to radiation in patients with stable angina compared with functional testing. ${ }^{12}$ However, no study has analysed the effectiveness of CT in a randomised comparison with coronary angiography. ${ }^{13}$

We investigated the comparative effectiveness of cardiac CT and coronary angiography in patients with suspected coronary artery disease and atypical angina or chest pain who were clinically referred for coronary angiography. We assessed the impact of CT on procedural complications, diagnostic yield of invasive coronary angiography, length of stay, radiation dose, long term clinical outcomes, and patient acceptance.

\section{Methods}

\section{Study design and patients}

This study (Coronary Artery Disease Management, CADMan) was a prospective randomised single centre clinical investigation of CT and coronary angiography. It was conducted and is reported in accordance with the Consolidated Standards of Reporting Trials (CONSORT) guidelines for non-drug trials. ${ }^{1415}$ From 18 February 2009 to 27 August 2015 we approached consecutive patients presenting during working days from $730 \mathrm{am}$ until 4 pm with suspected coronary artery disease and a clinical indication for coronary angiography on the basis of atypical presentation for study participation. Atypical presentation was defined as the presence of a maximum of two of the three criteria for typical angina pectoris (retrosternal chest discomfort, precipitation by exertion, and prompt relief within 30 seconds to $10 \mathrm{~min}$ utes by rest or nitroglycerine) using a clinically relevant classification of chest discomfort. ${ }^{16}$ We used the Duke clinical score to assess the pretest probability of coronary artery disease. ${ }^{17}$ Patients were excluded according to the study protocol (see appendix A) if they had two positive test results for ischaemia, were not in sinus rhythm, had signs of myocardial infarction (persistent ST segment elevation, creatine phosphokinaseMB > $24 \mathrm{U} / \mathrm{L}$, or pulmonary oedema due to ischaemia), refused or were incapable of providing informed consent, could not hold their breath for five seconds, were aged less than 30 years, or had a history of or were receiving dialysis.

\section{Study procedures}

We randomly assigned patients with suspected coronary artery disease to CT, followed by coronary angiography if positive for obstructive coronary artery disease, or to direct coronary angiography. In both tests, obstructive coronary artery disease was defined as at least one $50 \%$ diameter stenosis in the left main coronary artery or at least one $70 \%$ diameter stenosis in other coronary arteries. Stenosis diameters were assessed as described. ${ }^{18}$ Patients with suspected obstructive coronary artery disease in the CT group subsequently underwent late enhancement magnetic resonance imaging as described ${ }^{19}$ to identify those with more than 50\% transmural extent of non-viable myocardium in the area supplied by a stenosed artery and thus considered unlikely to benefit from revascularisation. ${ }^{20}$ Patients with non-calcified coronary plaques on CT were recommended for intensified risk factor modification and statin treatment ${ }^{21}$ as this management might reduce cardiovascular death and myocardial infarction. ${ }^{22}$ Patients randomised to coronary angiography underwent this test according to clinical practice at our institution, and the findings guided subsequent guideline based management. ${ }^{23}$

\section{CT and coronary angiography protocol}

Cardiac CT was performed and evaluated as described. ${ }^{24}$ Images were evaluated by two independent readers, of whom at least one was a board certified radiologist (MD, $\mathrm{MR}, \mathrm{EZ}$ ) and, as in routine clinical practice, decisions were made by consensus. In patients with heart rates up to $65 \mathrm{bpm}, 320$ row coronary CT angiography enabled reconstruction windows of $175 \mathrm{msec}$ (Aquilion ONE, Toshiba Medical Systems, 121 patients) or 137.5 msec (Aquilion ONE Vision Edition, 44 patients). In patients with heart rates of at least $65 \mathrm{bpm}$, temporal resolution was improved by two beat acquisition in 23 patients and three beat acquisition in two patients. ${ }^{25}$ Coronary calcium scoring was used to individually adjust the acquisition length of CT angiography, ${ }^{2627}$ but was not used to defer CT angiography because up to $19 \%$ of patients with symptoms and zero calcium scores might have obstructive coronary artery disease. ${ }^{28}$ Coronary angiography was performed as in clinical practice in our institution after hospital admission. A vascular closure device was used in 64 of the 186 coronary angiographies.

\section{Primary and secondary outcomes}

The planned primary outcome was major procedural complications occurring within 48 hours of the last procedure related to CT or to coronary angiography and analysis was at individual patient level. The occurrence of procedural complications was checked during visits 24 and 48 hours after the last related procedure. Major procedural complications were a composite endpoint and included death, stroke, myocardial infarction, or other major complications prolonging the in-hospital stay by at least 24 hours. Minor procedural complications occurring within 48 hours of the last procedure related to CT or coronary angiography did not fulfil these criteria and were added to the comparison. A planned secondary outcome was comparison of length of stay between the two groups. We also assessed overall exposure to radiation in both study groups as a planned secondary outcome using dose-length products and dose-area products, which were converted using factors of $0.017 \mathrm{mSv} / \mathrm{mGy} \times \mathrm{cm}$ for $\mathrm{CT}^{29}$ and $0.22 \mathrm{mSv} / \mathrm{cGy} \times \mathrm{cm}^{2}$ for coronary angiography. ${ }^{30}$ Radiation dose in the 
coronary angiography group included dose of percutaneous coronary intervention if done. Radiation dose in the CT group included the effective dose of CT and coronary angiography and/or percutaneous coronary intervention if performed. In addition, we evaluated the diagnostic yield of coronary angiography, defined as the proportion of obstructive coronary artery disease found in both groups by coronary angiography, and we added the reduction in need for coronary angiography in the CT group over time-with a maximum follow-up to 16 August 2016-to the analysis on request during the review process. As a secondary outcome, long term clinical events (major adverse cardiovascular events (MACE), defined as a composite endpoint of myocardial infarction, cardiac death, stroke, unstable angina pectoris, or re-revascularisation or first revascularisation) were recorded with a maximum follow-up to 16 August 2016. Re-revascularisation or first revascularisation was recorded only if occurring more than two months after randomisation. ${ }^{31}$ We obtained patients' acceptance and preference of the procedures using a previously validated questionnaire. ${ }^{32}$ See appendix A for details of the study protocol, including all planned primary and secondary objectives and changes to the study protocol.

\section{Randomisation and outcome assessment}

Patients were randomised to CT or coronary angiography if a clinical referral to coronary angiography and all inclusion criteria were met. Randomisation was by a computer generated list (nQuery 7.0; Statistical Solutions, Cork, Republic of Ireland) using sequentially numbered, opaque and sealed envelopes, which were opened just before allocation to the intervention. ${ }^{14}$ Investigators and participants were blinded to the randomisation sequence but could not be blinded to the assigned group because of the apparent differences between CT and coronary angiography. Independent investigators who were blinded to the randomisation group and were not part of the study team anonymously assessed outcomes.

\section{Statistical analysis}

As outlined in chapter 11 of the study protocol, we expected procedural complications of $15 \%$ in the coronary angiography group and 5\% in the CT group (see appendix A). We considered a reduction in procedural complications by a factor of three to be relevant from a clinical perspective. Overall, we needed 160 patients in each group to detect these differences, with $80 \%$ power at a two sided $\alpha$ significance level of 0.05 using a $\chi^{2}$ test with correction for continuity (nQuery Advisor 7.0, Statistical Solutions, Cork, Republic of Ireland). With a conservatively estimated drop-out rate of $5 \%$, we randomised 340 patients.

Using a $\chi^{2}$ test we evaluated the primary outcome, procedural complications, in the entire intention to treat population, retaining participants in their originally assigned groups after excluding those who withdrew consent after randomisation. Secondary outcomes were evaluated using $\chi^{2}$ or t tests; length of stay and exposure to radiation were compared using a Wilcoxon rank-sum test; time to first MACE during long term follow-up was analysed using the Cox proportional-hazards model; ${ }^{33}$ and the need for, possibly multiple, coronary angiography over time was compared using Poisson regression with offset observation time. We performed all comparisons using two sided significance tests; SPSS software, version 20.0, and SAS software, version 9.4 (Poisson regression) was used for statistical analysis.

\section{Patient involvement}

No patients were involved in setting the research question or the outcome measure, nor were they involved in developing plans for design or implementation of the study. All patients included in the study were asked how they experienced the diagnostic tests and to make suggestions about further improvements. The results of the research will be reported to the study participants, and the DISCHARGE (Diagnostic Imaging Strategies for Patients with Stable Chest Pain and Intermediate Risk of Coronary Artery Disease: Comparative Effectiveness Research of Existing Technologies) trial team ${ }^{34}$ will consider them in planning the analysis of this multicentre effort together with patient representatives at all sites.

\section{Results}

\section{Study population}

During the study period, 739 patients with suspected coronary artery disease and an indication for coronary angiography based on atypical angina or chest pain were referred to our centre and were potentially eligible for study participation (fig 1). A total of 144 patients were excluded, mostly because of two positive test results for ischaemia (67 patients) and atrial fibrillation (32 patients). The prevalence of coronary artery disease among the 144 patients with exclusion criteria was higher than in the final study population (see supplementary table 1 in appendix B). Overall, 255 of the 549 eligible patients were not randomised, mainly because of exclusion by clinicians ( $\mathrm{n}=144)$, logistical problems $(n=45)$, and patients declining to give consent $(n=37)$. The prevalence of coronary artery disease among the 255 eligible patients not randomised was not significantly different from the final study population (see supplementary table 1 in appendix B). Overall, 340 patients with a pretest probability of coronary artery disease of $34.6 \%$ (SD 23.5\%) were randomised to CT or coronary angiography (fig 1). Of the 168 patients assigned to the CT group, one withdrew informed consent and two underwent coronary angiography per clinician request. Ten of the 172 patients randomised to coronary angiography withdrew informed consent for participation in the study. Altogether, 167 and 162 patients with complete follow-up 48 hours after the last procedure related to CT or coronary angiography were available for the primary outcome analysis in the CT and coronary angiography group, respectively (fig 1).

\section{Patient characteristics}

Patients had a mean age of 60.4 years (SD 11.4 years) and 50\% were women (table 1). Most patients had 
through the study.

$\mathrm{ECG}=$ electrocardiography;

$M R I=$ magnetic resonance

imaging; $\mathrm{CT}=$ computed tomography

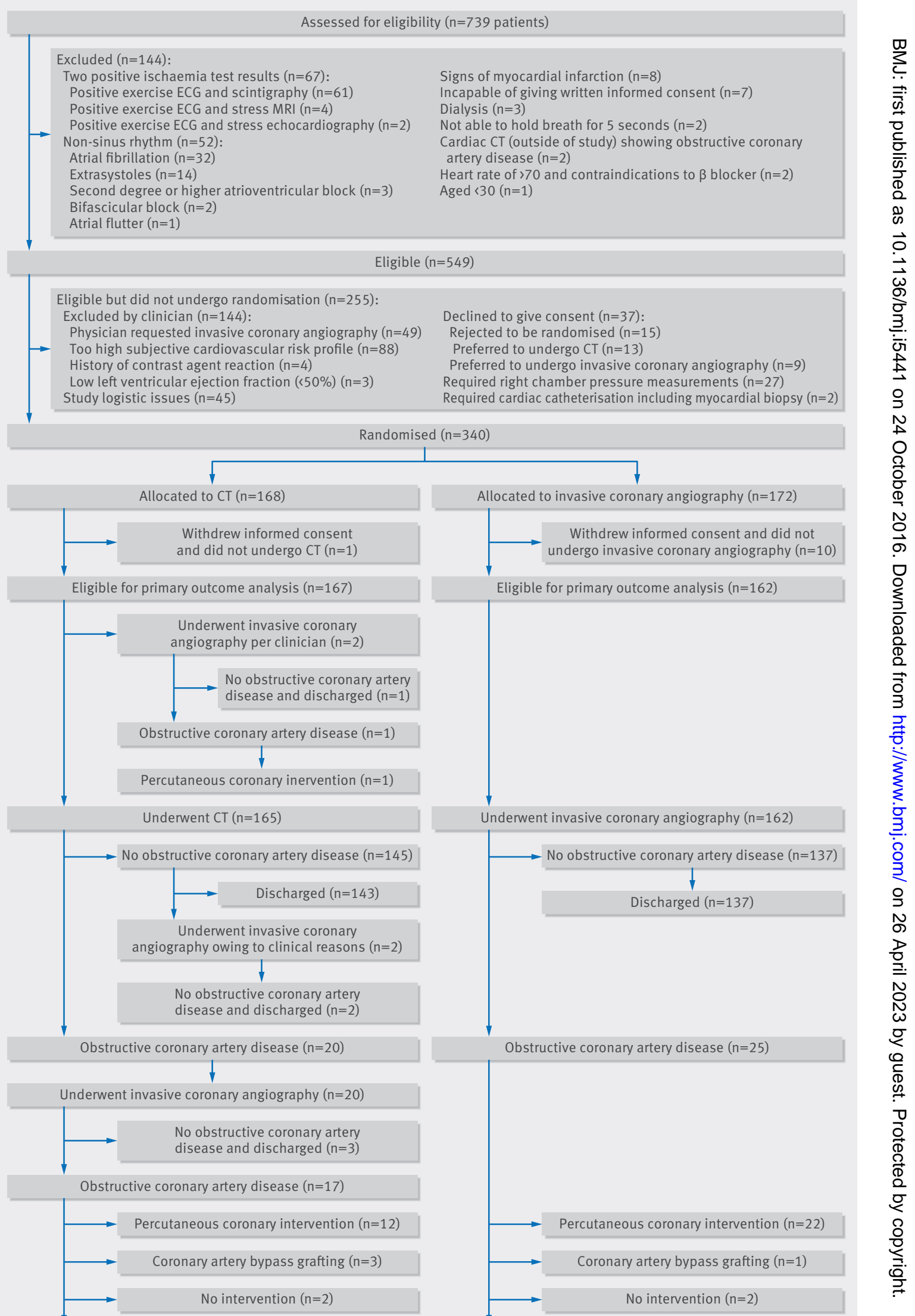

Complete follow-up for procedural complications related to any diagnostic or therapeutic procedure in this group $(n=167)$
Signs of myocardial infarction $(\mathrm{n}=8)$

Dialysis $(n=3)$

Not able to hold breath for 5 seconds $(n=2)$

Cardiac CT (outside of study) showing obstructive coronary

Second degree or higher atrioventricular block $(n=3)$

Bifascicular block $(n=2)$

Aged $<30(n=1)$

Required right chamber pressure measurements ( $n=27$ )

Low left ventricular ejection fraction $(450 \%)(n=3)$

Required cardiac cathere 
Table 1 | Baseline characteristics of patients with an intermediate probability of coronary artery disease assigned to computed tomography or coronary angiography. Values are means (SDs) unless stated otherwise*

\begin{tabular}{|c|c|c|}
\hline Characteristics & Computed tomography $(n=167)$ & Coronary angiography $(n=162)$ \\
\hline Age (years) & $60.4(11.3)$ & $60.4(11.4)$ \\
\hline No (\%) of women & $88(52.7)$ & $78(48.1)$ \\
\hline \multicolumn{3}{|l|}{ No (\%) with atypical chest discomforttł: } \\
\hline Atypical angina & 65 (38.9) & 79 (48.8) \\
\hline Non-anginal chest pain & $97(58.1)$ & $80(49.4)$ \\
\hline Other chest discomfort & $5(3.0)$ & $3(1.8)$ \\
\hline No (\%) with discomfort relief by nitroglycerine or rest & $20(12.0)$ & $29(18.0)$ \\
\hline Pretest probability of coronary artery disease: & $31.3(21.1)$ & $37.3(24.8)$ \\
\hline No (\%) with pretest probability of coronary artery disease $<10 \%$ & $19(11.4)$ & $25(15.4)$ \\
\hline Coronary artery disease by final examination & $1(5.3)$ & $1(4.0)$ \\
\hline No (\%) with pretest probability of coronary artery disease $10-60 \%$ & $124(74.2)$ & $98(60.5)$ \\
\hline Coronary artery disease by final examination & $9(7.2)$ & $11(11.2)$ \\
\hline No (\%) with pretest probability of coronary artery disease $>60 \%$ & $24(14.4)$ & $39(24.1)$ \\
\hline Coronary artery disease by final examination & $8(33.3)$ & $13(33.3)$ \\
\hline \multicolumn{3}{|l|}{ Hospital admission status at time of randomisation: } \\
\hline No (\%) of inpatients & $92(55.1)$ & $94(58.0)$ \\
\hline No (\%) of outpatients & 75 (44.9) & $68(42.0)$ \\
\hline \multicolumn{3}{|l|}{ Functional tests within 6 months before randomisation: } \\
\hline No (\%) of positive functional test before randomisation & $43(25.7)$ & $45(27.7)$ \\
\hline Coronary artery disease by final examination & $6(14.0)$ & $9(20.0)$ \\
\hline No (\%) of positive exercise electrocardiograms & $33(76.7)$ & $40(88.8)$ \\
\hline Coronary artery disease by final examination & $5(15.2)$ & $9(22.5)$ \\
\hline No (\%) of positive single photon emission CTs & $7(16.3)$ & $2(4.4)$ \\
\hline Coronary artery disease by final examination & $0(0)$ & $0(0)$ \\
\hline No (\%) of positive stress echocardiographies & $3(7.0)$ & $3(6.6)$ \\
\hline Coronary artery disease by final examination & $1(33.3)$ & $0(0)$ \\
\hline No (\%) of negative functional tests before randomisation & $41(24.5)$ & $47(29.0)$ \\
\hline Coronary artery disease by final examination & $4(9.7)$ & $4(8.5)$ \\
\hline No (\%) of negative exercise electrocardiograms & $34(82.9)$ & $40(85.1)$ \\
\hline Coronary artery disease by final examination & $4(11.8)$ & $3(7.5)$ \\
\hline No (\%) of negative single photon emission CTs & $2(4.9)$ & $4(8.5)$ \\
\hline Coronary artery disease by final examination & $0(0)$ & $0(0)$ \\
\hline No (\%) of negative stress echocardiographies & $5(12.2)$ & $6(12.8)$ \\
\hline Coronary artery disease by final examination & 0 & $1(16.6)$ \\
\hline No (\%) without functional test before randomisation & $83(49.7)$ & $70(43.2)$ \\
\hline Coronary artery disease by final examination & $8(9.6)$ & $12(17.1)$ \\
\hline \multicolumn{3}{|l|}{ Cardiovascular risk factors: } \\
\hline Body mass index & $27.5(4.7)$ & $27.0(4.6)$ \\
\hline No (\%) of smokers & $41(24.5)$ & $34(21.0)$ \\
\hline No (\%) of former smokers & $47(28.1)$ & $51(31.5)$ \\
\hline Average pack years for smokers and former smokers & $19.5(14.0)$ & $20.0(17.1)$ \\
\hline No (\%) with hyperlipidaemia & $95(56.9)$ & $81(51.0)$ \\
\hline No (\%) with arterial hypertension & $111(66.5)$ & $112(69.1)$ \\
\hline No (\%) with diabetes mellitus & $15(9.0)$ & $30(18.5)$ \\
\hline \multicolumn{3}{|l|}{ No (\%) with cardiovascular drugs: } \\
\hline Chronic statin intake & $42(25.1)$ & $41(25.3)$ \\
\hline Acetylsalicylic acid intake & $47(28.1)$ & $41(25.3)$ \\
\hline Chronic $\beta$ blocker intake & $70(41.9)$ & $71(43.8)$ \\
\hline Insulin treatment & $3(1.8)$ & $3(1.8)$ \\
\hline Oral antidiabetes treatment & $11(6.6)$ & $17(10.5)$ \\
\hline \multicolumn{3}{|l|}{ No (\%) with cardiovascular medical history: } \\
\hline Family history of premature coronary artery disease§ & $24(14.4)$ & $16(9.9)$ \\
\hline Stroke & $8(4.8)$ & $7(4.3)$ \\
\hline Peripheral artery disease & $2(1.2)$ & $0(0)$ \\
\hline Carotid artery disease & $9(5.4)$ & $10(6.2)$ \\
\hline
\end{tabular}

*Proportions might not total $100 \%$ owing to rounding. The only notable difference between the groups was for pretest probability, which was higher in the coronary angiography group, and for diabetes, which was more common in the coronary angiography group.

tAtypical chest discomfort was an inclusion criterion and was defined as presence of a maximum of two of three criteria for typical angina pectoris (retrosternal chest discomfort, precipitation by exertion, and prompt relief within 30 seconds to 10 minutes by rest or nitroglycerine) as defined by Diamond. ${ }^{16}$ Therefore, patients with atypical angina (two of three criteria), non-anginal chest pain (one of three criteria), or other chest discomfort (no criteria) could be included. fUnstable angina ${ }^{35}$ was not present in any patient with atypical presentation.

§Premature coronary artery disease was considered if a first degree relative, before age 55 years in male relatives and before 65 years in female relatives, experienced a fatal or non-fatal myocardial infarction and/or coronary angioplasty/coronary artery bypass surgery. 
moderate cardiovascular risk profiles and either atypical angina pectoris or non-anginal chest pain. Among the 329 patients available for analysis, the prevalence of obstructive coronary artery disease defined by the last examination was $13 \%$ (fig 1), which was relevantly lower than suggested by the 34.3\% (SD 23.4\%) pretest probability. The true positive rate of functional tests performed within six months before randomisation was $14 \%$ and $20 \%$ (table 1 ). In the CT group, coronary angiography was used for determining prevalence in 24 patients. All CT examinations were deemed interpretable by both readers. Magnetic resonance imaging was conducted before coronary angiography in 17 of the 20 patients with a diagnosis of obstructive coronary artery disease by CT, whereas three patients underwent coronary angiography directly owing to metal implants $(n=1)$ or claustrophobia $(n=2)$. The 17 patients who underwent magnetic resonance imaging had at least $50 \%$ viable tissue in myocardial regions supplied by stenotic coronary arteries so that coronary angiography was not deferred. The median interval between CT and coronary angiography in the 22 patients who underwent both procedures (fig 1 ) was 47 hours (interquartile range 18.7-91.6). Obstructive coronary artery disease was excluded in $88.6 \%$ of patients in the CT group (149 of 167$)$ and $84.6 \%$ (137 of 162) in the coronary angiography group ( $\mathrm{P}=0.28$; fig 1$)$.

\section{Primary and secondary outcomes}

Major procedural complications occurring within 48 hours of the last procedure related to CT or coronary angiography were uncommon $(0.3 \%)$ and similar across groups (table 2). In general, both CT and coronary angiography were safe procedures with no deaths or strokes. Only one myocardial infarction occurred in the CT group after percutaneous coronary intervention (table 2). Minor procedural complications were less common in the CT group than coronary angiography group (3.6\% v 10.5\%, P=0.014; table 2). Most of the 23 minor procedural complications were related to the puncture site of coronary angiography (haematoma or secondary bleedings) and only two were consequences

\begin{tabular}{|c|c|c|c|}
\hline Outcomes & $\begin{array}{l}\text { Computed } \\
\text { tomography } \\
(\mathrm{n}=167)\end{array}$ & $\begin{array}{l}\text { Coronary } \\
\text { angiography } \\
(n=162)\end{array}$ & P value \\
\hline Major procedural complicationst: & $1(0.6)$ & $0(0)$ & 1.00 \\
\hline Myocardial infarction & $1(0.6)$ & $0(0)$ & 1.00 \\
\hline Death & $0(0)$ & $0(0)$ & \\
\hline Stroke & $0(0)$ & $0(0)$ & \\
\hline Other complications prolonging hospital stay by at least 24 hours & $0(0)$ & $0(0)$ & \\
\hline Minor procedural complicationsł: & $6(3.6)$ & $17(10.5)$ & 0.014 \\
\hline Haematoma at puncture site & $1(0.6)$ & $14(8.6)$ & $<0.001$ \\
\hline Secondary bleeding at puncture site & $1(0.6)$ & $1(0.6)$ & 1.00 \\
\hline Bradycardia & $2(1.2)$ & $0(0)$ & 0.50 \\
\hline Angina without infarction & $1(0.6)$ & $0(0)$ & 1.00 \\
\hline Allergoid reaction to contrast agent & $1(0.6)$ & $0(0)$ & 1.00 \\
\hline Stent migration & $0(0)$ & $1(0.6)$ & 0.49 \\
\hline Hypotension requiring treatment & $0(0)$ & $1(0.6)$ & 0.49 \\
\hline No (\%) of invasive coronary angiographies performed & $24(14)$ & $162(100)$ & $<0.001$ \\
\hline No/total No (\%) of invasive angiographies with obstructive coronary artery disease & $18 / 24(75)$ & 25/162 (15) & $<0.001$ \\
\hline $\begin{array}{l}\text { No/total No (\%) of invasive angiographies associated with interventional or surgical revascularisation of obstructive } \\
\text { coronary artery disease }\end{array}$ & $16 / 24(67)$ & 23/162 (14) & $<0.001$ \\
\hline Median (interquartile range) length of stay (hours) & $30.0(3.5-77.3)$ & $52.9(49.5-76.4)$ & $<0.001$ \\
\hline Median (interquartile range) radiation exposure (mSv)§ & $5.0(4.2-8.7)$ & $6.4(3.4-10.7)$ & 0.45 \\
\hline No (\%) of any event at long term follow-up I: & $7(4.2)$ & $6(3.7)$ & 0.86 \\
\hline Myocardial infarction & $1(0.6)$ & $0(0)$ & \\
\hline Cardiac death & $0(0)$ & $1(0.6)$ & \\
\hline Stroke & $0(0)$ & $1(0.6)$ & \\
\hline Unstable angina pectoris & $2(1.2)$ & $0(0)$ & \\
\hline Re-revascularisation or first revascularisation & $6(3.6)$ & $5(3.1)$ & 0.84 \\
\hline
\end{tabular}

*Proportions might not add to $100 \%$ owing to rounding.

tSingle procedure related myocardial infarction occurred after percutaneous coronary intervention in computed tomography (CT) group: during primary stent implantation, a dissection of the left anterior descending coronary artery occurred, leading to implantation of a second stent with over-stenting of a side branch. The patient developed mild angina, ST segment elevation of 0.1

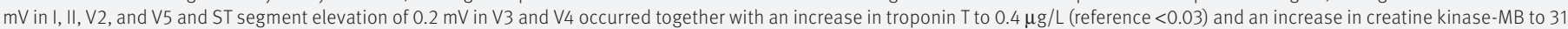
$\mathrm{U} / \mathrm{L}$ (reference <24) consistent with myocardial infarction 36

‡Of 23 minor procedural complications, six occurred in the CT group and 17 in the coronary angiography group ( $P=0.014)$. Of the minor procedural complications in the CT group, four occurred after coronary angiography and two after CT. Haematoma at the puncture site was not associated with access (femoral $v$ radial, $P=0.782$ ) and was not significantly reduced by use of a vascular closure device in this non-randomised comparison $(\mathrm{P}=0.06$ ). Femoral access was most common (175 of the 186 patients who finally underwent coronary angiography) and radial access was used in the remaining 11 patients. Complications after $\mathrm{CT}$ included one allergoid reaction to contrast agent with pruritus and skin rash, which was successfully treated with prednisone and $\mathrm{H} 1$ antihistamine, and bradycardia with hypotension after $\beta$ blockade for $\mathrm{CT}$, which was treated with saline infusion.

$\S 0 v e r a l l$ median radiation dose included coronary angiographies and revascularisations in both groups.

१Hazards ratios for any event and revascularisation were 0.90 ( $95 \%$ confidence interval 0.30 to 2.69 ) and 0.89 ( 0.27 to 2.90 ), respectively. For small number events no P values and hazards

ratios of Cox model are given. 
of CT (table 2). Cardiac CT compared with direct coronary angiography, reduced the need for coronary angiography from $100 \%$ to $14 \%$ (95\% confidence interval $9 \%$ to $20 \%, \mathrm{P}<0.001$ ) and was associated with a significantly greater diagnostic yield of coronary angiography, defined as the proportion of coronary angiographies showing obstructive coronary artery disease $(75 \% \mathrm{v}$ $15 \%, \mathrm{P}<0.001$; table 2). Also, the proportion of coronary angiographies associated with revascularisation of obstructive coronary artery disease was significantly larger in the CT group than coronary angiography group $(67 \%$ v 14\%, $\mathrm{P}<0.001$; table 2).

\section{Length of stay and radiation exposure}

Cardiac CT shortened the median length of stay in the coronary angiography group from 52.9 hours (interquartile range 49.5-76.4) to 30.0 hours (3.5-77.3, $\mathrm{P}<0.001$; fig 2). In the group of inpatients at the time of randomisation, the length of stay was not significantly reduced by CT (60.3 hours; interquartile range 32.6-98.5 v 68.0 hours; 50.2-105.8, $\mathrm{P}=0.12$ ). Overall median exposure to radiation, including coronary angiography and revascularisations, was similar between the CT group (5.0 $\mathrm{mSv}$; interquartile range 4.2-8.7) and coronary angiography group (6.4 $\mathrm{mSv}$; 3.4-10.7, $\mathrm{P}=0.45$; fig 3 ). Also, the distribution of radiation exposure was right skewed in both groups (see supplementary figure 1 in appendix B). Adding percutaneous coronary intervention to coronary angiography led to a significantly increased median effective radiation dose (see supplementary figure 2 in appendix B).

Reduction in need for coronary angiography over time The reduction in need for coronary angiography in the CT group did not decline over time (fig 4), and there was a significantly lower rate of coronary angiographies per patient during the first six months after randomisation to the CT group compared with the coronary angiography group $(0.25 v 1.1, \mathrm{P}<0.001)$. The cumulative relative risk adjusted for observation time for coronary angiography in the coronary angiography versus CT group was 4.2 (95\% confidence interval 3.0 to $5.8, \mathrm{P}<0.001$ ) in the first six months and 3.1 (2.3 to $4.0, \mathrm{P}<0.001$ ) for the entire observation period. In the second six months after randomisation, the rate of coronary angiographies per patient did not differ between the CT group and coronary angiography group $(0.027 v 0.013$; fig 4 , relative risk $0.78, \mathrm{P}=0.74$ ), and from the second year of observation the relative risk was $0.81(\mathrm{P}=0.56)$.

\section{Long term clinical outcomes}

After a median follow-up of 3.3 years (interquartile range 1.3-4.6 years), MACE had occurred in seven of 167 patients in the CT group (4.2\%) and six of $162(3.7 \%)$ in the coronary angiography group (adjusted hazard ratio $0.90,95 \%$ confidence interval 0.30 to $2.69, \mathrm{P}=0.86$ ). First revascularisation and re-revascularisation, at least two months after randomisation, occurred in six of 167 patients in the CT group (3.6\%) and five of 162 (3.1\%) in the coronary angiography group (adjusted hazard ratio $0.89,0.27$ to $2.90, \mathrm{P}=0.84$; table 2 ).

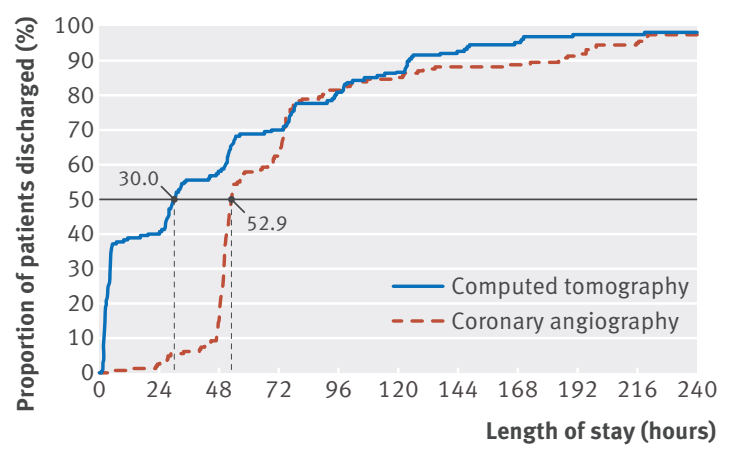

No of patients for outpatient evaluation or in hospital Computed tomography $\begin{array}{lllllllllll}167 & 100 & 71 & 50 & 32 & 22 & 12 & 8 & 4 & 4 & 3\end{array}$ Coronary angiography $\begin{array}{lllllllllll}162 & 158 & 136 & 61 & 30 & 24 & 19 & 18 & 14 & 8 & 4\end{array}$

Fig 2 | Length of stay and proportion of patients discharged. Computed tomography (CT) shortened median length of stay from $\mathbf{5 2 . 9}$ hours in coronary angiography group (interquartile range 49.5-76.4) to 30.0 hours (3.5-77.3, $\mathrm{P}<0.001)$. Because coronary angiography in Germany is mostly done after hospital admission, patients in our study were mostly in hospital. Of the 167 patients in the CT group, 64 (38\%) were managed on an outpatient basis, $11(7 \%)$ had outpatient CT but were admitted to hospital for suspected coronary artery disease, and 92 (55\%) were randomised after hospital admission for clinically indicated coronary angiography

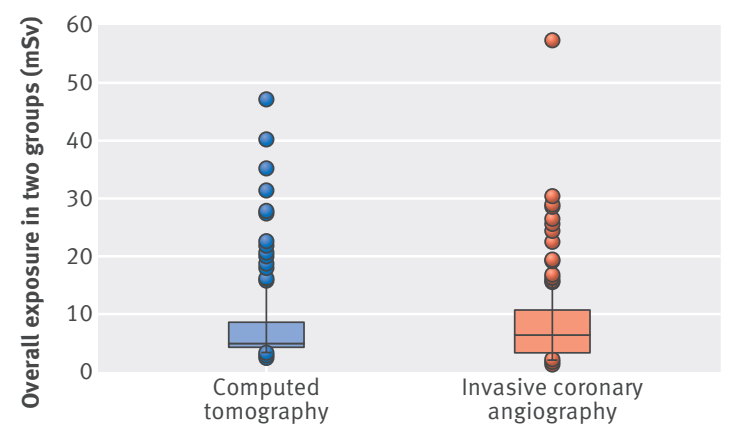

Fig 3 Exposure to radiation in computed tomography (CT) and invasive coronary angiography group. Box plots of radiation dose in both groups are shown. Median overall radiation exposure, including invasive angiographies and revascularisations, was similar between the CT group (5.0 $\mathrm{mSv}$; interquartile range 4.2-8.7) and coronary angiography group (6.4 mSv; 3.4-10.7, $\mathrm{P}=0.45)$. The dose for left ventriculography, which was done in 19 of the 24 patients who underwent coronary angiography in the CT group (79\%) and in 141 of the 162 patients in the coronary angiography ( $87 \%)$, was included in the radiation exposure of the respective group

\section{Patients' acceptance and preference}

Overall, 292 of the 329 patients (89\%) answered at least one question in the acceptance questionnaire. Approximately $79 \%$ of patients (219 of 278) stated that they would prefer CT for subsequent testing, whereas significantly fewer patients indicated preference for coronary angiography $(7 \% ; 20$ patients, $\mathrm{P}<0.001)$. Of the 20 patients who preferred coronary angiography, 18 underwent the procedure (17 without and one with 


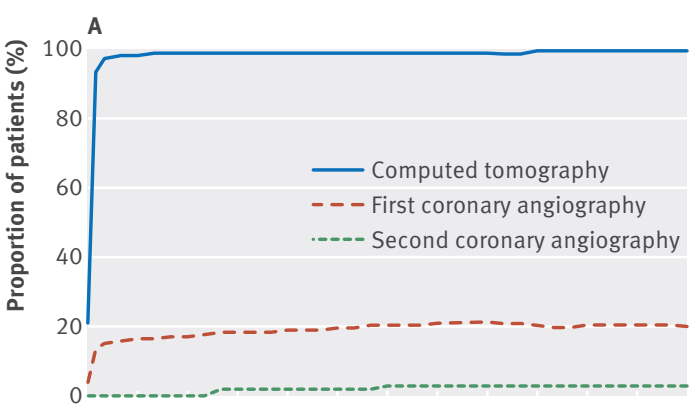

Follow-up data available

167159159159159159159158156154152152150

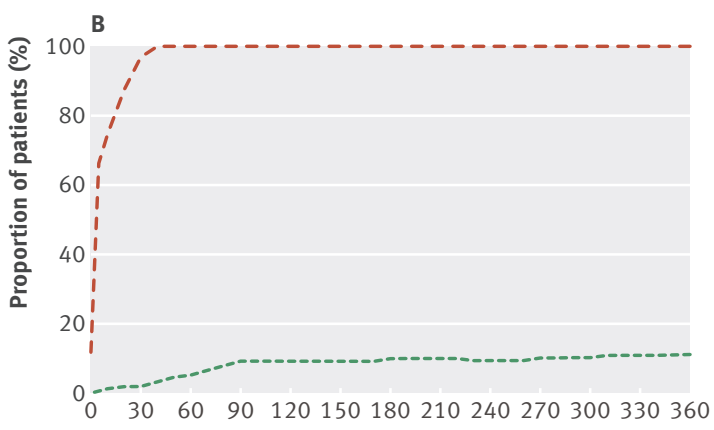

Follow-up data available

Time (days)

162153152152152152152150149148147146144

Fig 4 | Need for invasive coronary angiography over time. The rate of coronary angiographies was significantly lower per patient during the first six months after randomisation to the computed tomography (CT) group (A) compared with coronary angiography group $(0.25 v 1.1 ; \mathrm{B}, \mathrm{P}<0.001)$. In the 6-12 months after randomisation, the proportion of patients undergoing coronary angiography was the same between the groups $(P=0.74)$

percutaneous intervention) and two underwent CT. Preference for CT was higher in patients in the CT group (87\%; 127 of 146 patients) than in those in the coronary angiography group (70\%; 92 of 132 patients, $\mathrm{P}<0.001)$. Most patients who preferred CT did so because they would prefer non-invasive testing in the future $(n=79$; $36 \%$ ), and the implementation of this non-invasive testing should ideally result in a short examination time without the need for hospital admission ( $n=22 ; 10 \%)$. Among patients who preferred coronary angiography, $30 \%(n=6)$ did so because it is the established diagnostic procedure, and mostly because it enables subsequent minimally invasive treatment $(n=5 ; 25 \%)$.

\section{Discussion}

In this randomised study on the risks and benefits of a non-invasive versus invasive diagnostic test, patients with a clinical indication for coronary angiography because of atypical angina or chest pain suggestive of coronary artery disease were randomised to computed tomography (CT) or coronary angiography. The study addressed the important clinical question of whether invasive coronary angiography or CT should be performed in patients with a clinical indication for coronary angiography because of an intermediate probability of coronary artery disease. CT was found to be a safe gatekeeper for invasive coronary angiography, with no increase in long term events, and patients in the CT group had fewer minor but not major procedural complications compared with those who underwent direct coronary angiography. CT was associated with a five times greater diagnostic yield of coronary angiography in the CT group, defined as the proportion of coronary angiographies showing obstructive coronary artery disease, and reduced the need for invasive coronary angiography by sevenfold indicating the potential to reduce overuse of invasive procedures. ${ }^{37} 38$ The length of stay was significantly shortened with CT, whereas exposure to radiation was similar between the groups. Patient acceptance of non-invasive CT was better than acceptance of coronary angiography.

\section{Clinical context}

The significantly shorter length of stay in the CT group might help reduce costs when CT is used in properly selected patients with atypical presentation. 3940 Detailed analysis using data from the current and other randomised controlled trials ${ }^{9111241}$ could allow estimation of the relative cost effectiveness of CT versus invasive anatomical or functional tests for specific patient groups. ${ }^{42-44} \mathrm{CT}$ resulted in a similar radiation dose in the CT group and coronary angiography group. Personalised adjustment of X-ray tube output to individual patient sizes, and novel model based iterative reconstruction might further reduce the radiation dose of CT.45 In our study, overall radiation dose was reduced by individual adjustment of anatomical coverage of CT angiography using previous coronary calcium scanning. ${ }^{2627}$ The recent CRESCENT (Computed Tomography vs. Exercise Testing in Suspected Coronary Artery Disease) trial used a zero calcium score to exclude coronary artery disease and defer CT angiography, ${ }^{12}$ and our findings confirm the potential of this approach as only one of 71 patients with a zero calcium score had obstructive coronary artery disease (see supplementary table 2 in appendix B). Performing diagnostic tests also has psychologically mediated effects. In a randomised study, the investigators showed that even tests with no diagnostic value can reduce short term disability and increase satisfaction. ${ }^{46}$ Although the two tests performed in our study have diagnostic value, it is still likely that patients' expectations influence disability and preference, whereas procedural complications and events are unlikely to be affected. Importantly, more than three quarters of our patients indicated a preference for CT over coronary angiography for future imaging which, according to their assessment, should ideally be performed non-invasively, over a short period, and in an outpatient setting.

\section{Implementation issues}

CT is an accurate non-invasive alternative to coronary angiography. Its conduct is well standardised in guidelines ${ }^{47-49}$ and based on an international survey, ${ }^{50}$ and thus there is no barrier to implementation. However, easier access to CT might result in lower thresholds for 
non-invasive than for invasive imaging and could thereby increase subsequent invasive procedures. This undesired effect can be avoided if cardiac CT is carefully implemented and used in the right patients and at the right time. ${ }^{51}$ Identifying the right patients is not trivial in routine practice, and our study showed that clinical approaches to estimate pretest probability ${ }^{17}$ relevantly overestimate the true prevalence of coronary artery disease in patients with atypical angina or chest pain clinically referred for invasive coronary angiography. Therefore, optimised tools for predicting the probability of obstructive coronary artery disease are clinically desired.

\section{Strengths and limitations of this study}

This study was conducted at a single centre and thus the performance of CT may be different when conducted in routine clinical practice. Importantly, despite standardised implementation of CT technology in routine clinical practice, ${ }^{50}$ gaining sufficient expertise in cardiac CT takes at least 12 months..$^{5253}$ We are currently scaling up the study in a multicentric fashion in the DISCHARGE trial, and results are expected in 2020. The actual prevalence and revascularisation rates in the study were lower than assumed, resulting in largely overestimated rates for major procedural complications and an underpowered study for the predefined primary outcome, making the study inconclusive in this regard. In accordance with guidelines, most patients with suspected coronary artery disease referred for coronary angiography have typical presentation. ${ }^{123}$ This resulted in a long recruitment period of patients with atypical angina or chest pain referred for coronary angiography and in the interim might have resulted in changes to practice. In our population with atypical angina, no patient had more than 50\% transmural extent of non-viable myocardial tissue on late enhancement magnetic resonance imaging, which thus did not result in changes to management. Coronary angiography can be performed on an outpatient basis with shorter length of stay but testing this was not feasible in our university hospital setting, where also CT was mainly done after patients had been admitted. To counterbalance this, in the calculation for length of stay we included time needed for any outpatient CT.

\section{Comparison with other studies}

Our study shows that cardiac CT is a safe test to rule out coronary artery disease in patients with atypical angina or chest pain and that invasive coronary angiography is a safe procedure in patients with atypical presentation with an increase only in minor procedural complications, which is in line with a study in 61 patients. ${ }^{54}$ Importantly, CT increased the diagnostic yield of coronary angiography by a factor of five, indicating the potential to improve utilisation of resources. The diagnostic yield of $75 \%$ for coronary angiography in the CT group was similar to that in the National Cardiovascular Data Registry and higher than reported for functional tests (approximately 45\%). ${ }^{55}$ CT may thus be able to act as an effective gatekeeper to the catheterisation laboratory ${ }^{56}$ and increase the reported low diagnostic yield of coronary angiography. ${ }^{2}$ Greater doctor confidence in CT findings was reported in the SCOTHeart trial, which compared CT with standard of care including functional tests. ${ }^{11}$ Our study adds to this by showing low true positive and high true negative rates of functional tests performed within six months before randomisation. Thus, CT might be able to replace functional testing in certain patients. Our prospective study also adds to these findings by showing a reduction of unnecessary invasive coronary angiographies by CT in patients with atypical presentation referred for coronary angiography.

\section{Conclusion}

The search is ongoing for a gatekeeper that will reliably exclude normal invasive coronary angiography. This randomised trial showed that in patients with atypical symptoms suggestive of coronary artery disease requiring coronary angiography, CT safely deferred invasive coronary angiography with no increase in long term events, reduced minor but not major procedural complications compared with direct coronary angiography, and shortened the length of hospital stay. CT was associated with reduced coronary angiography rates and increased the diagnostic yield of this procedure but did not reduce exposure to radiation. More patients preferred the coronary CT first approach.

We thank all the participants; radiographic staff; referring physicians; Gert Baumann (retired chair, Department of Cardiology); Lars Brillat, Mark Beling, Jürgen Frille, Angelika Frille, Fabian Stenzel, Lisa Hartmann, Robert Haase, Daniel Preuß, Paolo Ibes, Andrea Marek, Wasiem Sanad, Thomas Frank, and Ralf Offermann, for their collaboration; Jürgen Scholze, Karl Stangl, Verena Stangl, and Yvonne Dörffel for the management of the patients; Bettina Herwig for initial copy editing; Maria Bosserdt, Hans Tepe, and Laura Elzenbeck for data management; and Wolfgang Rutsch (retired chair of our catheterisation laboratory) for his vision and support. The data safety and monitoring board: Albert de Roos (radiologist, Leiden University, Netherlands), Thomas Meinertz (cardiologist, Hamburg, Germany), and Andreas Faldum (statistician, Münster, Germany) independently reviewed all potential anonymised major and minor procedural complications and major adverse cardiovascular events blinded to the study group.

Contributors: MD had the idea for the study. MD, PM, ML, and EZ designed the study. MR and PS helped in the design of the study. MD drafted the manuscript and all other authors suggested revisions of important intellectual content. MD, MR, PM, BK, SF, HD, SP, PS, MB, BH, $E S, M L$, and $E Z$ analysed and interpreted the data, and revised the manuscript for important intellectual content. EZ and MD had equal contributions. MD is the guarantor.

Funding: This study was funded by a grant of the Heisenberg programme of the German Research Foundation to Marc Dewey. All researchers are independent of the funding bodies. The funding bodies had no role in the study design; in the collection, analysis and interpretation of data; in the writing of the report; and in the decision to submit the manuscript for publication.

Competing interests: All authors have completed the ICMJE uniform disclosure form at www.icmje.org/coi-disclosure.pdf (available on request from the corresponding author) and declare that (1) MD has support from the Heisenberg programme of the German Research Foundation for the submitted work; (2) MD has relationships with Bayer, Bracco, Cardiac MR Academy, European Commission, European Regional Development Fund, German Foundation of Heart Research, German Federal Ministry of Education and Research, GE Healthcare, Guerbet, Springer, and Toshiba; BH has relationships with Bayer, Bracco, GE, Guerbet, Philips, Siemens, and Toshiba; (3) their spouses, partners, or children have no financial relationships that may be relevant to the submitted work; and (4) have no non-financial interests 
that may be relevant to the submitted work. Institutional master research agreements exist with Siemens Medical Solutions, Philips Medical Systems, and Toshiba Medical Systems. The terms of these arrangements are managed by the legal department of CharitéUniversitätsmedizin Berlin.

Ethical approval: This study was approved by the local research ethics committee of Charité (EA-1-080-08) and the German Federal Office for Radiation Protection (Z5-22462/2-2008-048). All patients gave written informed consent before randomisation.

Data sharing: Requests for patient level data will be considered by the CAD-Man trial group.

Transparency: The lead author (MD) affirms that the manuscript is an honest, accurate, and transparent account of the study being reported; that no important aspects of the study have been omitted; and that any discrepancies from the study as planned (and, if relevant, registered) have been explained.

This is an Open Access article distributed in accordance with the Creative Commons Attribution Non Commercial (CC BY-NC 3.0) license, which permits others to distribute, remix, adapt, build upon this work non-commercially, and license their derivative works on different terms, provided the original work is properly cited and the use is non-commercial. See: http://creativecommons.org/licenses/ by-nc/3.0/

1 Montalescot G, Sechtem U, Achenbach S, et al. Task Force Members ESC Committee for Practice Guidelines Documen Reviewers. 2013 ESC guidelines on the management of stable coronary artery disease: the Task Force on the management of stable coronary artery disease of the European Society of Cardiology. Eur Heart J 2013;34:2949-3003. doi:10.1093/eurheartj/ eht296.

2 Patel MR, Peterson ED, Dai D, et al. Low diagnostic yield of elective coronary angiography. N Engl J Med 2010;362:886-95doi:10.1056/ NEJMoa0907272.

3 Moschovitis A, CookS, Meier B. Percutaneous coronary interventions in Europe in 2006. Eurolntervention 2010;6:189-94. doi:10.4244/ EIJV6I2A31.

4 Noto TJ Jr, , Johnson LW, Krone R, et al. Cardiac catheterization 1990: a report of the Registry of the Society for Cardiac Angiography and Interventions (SCA\&I). Cathet Cardiovasc Diagn 1991;24:75-83. doi:10.1002/ccd.1810240202.

5 Levenson B, Albrecht A, Göhring S, et al. QulK-Register des Bundesverbandes Niedergelassener Kardiologen (BNK). [6th report of the German Association of Cardiologists in private practice (BNK) on quality assurance in cardiac catheterization and coronary intervention 2006-2009]. Herz 2011:36:41-9. doi:10.1007/s00059-011-3423-x.

6 Schuetz GM, Schlattmann P, Dewey M. Use of $3 \times 2$ tables with an intention to diagnose approach to assess clinical performance of diagnostic tests: meta-analytical evaluation of coronary CT angiography studies. BMJ 2012;345:e6717. doi:10.1136/bmj.e6717.

7 Schlattmann P, Schuetz GM, Dewey M. Influence of coronary artery disease prevalence on predictive values of coronary $\mathrm{CT}$ angiography: a meta-regression analysis. Eur Radiol 2011;21:1904-13. doi:10.1007/ s00330-011-2142-2.

8 Genders TS, Petersen SE, Pugliese F, et al. The optimal imaging strategy for patients with stable chest pain: a cost-effectiveness analysis. Ann Intern Med 2015;162:474-84. doi:10.7326/M14-0027.

9 Douglas PS, Hoffmann U, Patel MR, et al. PROMISE Investigators. Outcomes of anatomical versus functional testing for coronary artery disease. N Engl/ Med 2015:372:1291-300. doi:10.1056/NEIMoa 1415516.

10 Newby DE, Fox KA, Flint LL, Boon NA. A 'same day' direct-access chest pain clinic: improved management and reduced hospitalization. QJM 1998;91:333-7. doi:10.1093/qjmed/91.5.333

11 SCOT-HEART investigators. CT coronary angiography in patients with suspected angina due to coronary heart disease (SCOT-HEART): an open-label, parallel-group, multicentre trial. Lancet 2015;385:238391. doi:10.1016/S0140-6736(15)60291-4.

12 Lubbers M, Dedic A, Coenen A, et al. Calcium imaging and selective computed tomography angiography in comparison to functional testing for suspected coronary artery disease: the multicentre, randomized CRESCENT trial. Eur Heart/ 2016;37:1232-43. doi:10.1093/eurheartj/ehv700.

13 Schoenhagen P, Dewey M. CT assessment of coronary artery disease: trends and clinical implications. JACC Cardiovasc Imaging 2013;6:1072-4. doi:10.1016/i.jcmg.2013.07.006.

14 Moher D, Hopewell S, Schulz KF, et al. CONSORT 2010 explanation and elaboration: updated guidelines for reporting parallel group randomised trials. BMJ 2010;340:c869. doi:10.1136/bmj.c869.

15 Schulz KF, Altman DG, Moher D, Group C. CONSORT Group. CONSORT 2010 statement: updated guidelines for reporting parallel group randomised trials. BMJ 2010;340:c332. doi:10.1136/ bmi.c332.
16 Diamond GA. A clinically relevant classification of chest discomfort J Am Coll Cardiol 1983;1:574-5. doi:10.1016/S0735-1097(83) 80093-X.

17 Pryor DB, Shaw L, McCants CB, et al. Value of the history and physical in identifying patients at increased risk for coronary artery disease. Ann Intern Med 1993;118:81-90. doi:10.7326/0003-4819-118-2-19930115000001.

18 Dewey M, Rutsch W, Schnapauff D, Teige F, Hamm B. Coronary artery stenosis quantification using multislice computed tomography. Invest Radiol 2007:42:78-84. doi:10.1097/01.rli.0000251569. 01317.60 .

19 Simonetti OP, Kim RJ, Fieno DS, et al. An improved MR imaging technique for the visualization of myocardial infarction. Radiology 2001;218:215-23. doi:10.1148/radiology.218.1.r01ja50215.

20 Kim RJ, Wu E, Rafael A, et al. The use of contrast-enhanced magnetic resonance imaging to identify reversible myocardial dysfunction. N Engl I Med 2000:343:1445-53, doi:10.1056/NEIM2000111634 32003.

21 Perk J, De Backer G, Gohlke H, et al. European Guidelines on cardiovascular disease prevention in clinical practice (version 2012) The Fifth Joint Task Force of the European Society of Cardiology and Other Societies on Cardiovascular Disease Prevention in Clinical Practice (constituted by representatives of nine societies and by invited experts). Developed with the special contribution of the European Association for Cardiovascular Prevention \& Rehabilitation (EACPR). Eur Heart J 2012;33:1635-701. doi:10.1093/eurheartj/ ehs092.

22 Chow BJ, Small G, Yam Y, et al. CONFIRM Investigators. Prognostic and therapeutic implications of statin and aspirin therapy in individuals with nonobstructive coronary artery disease: results from the CONFIRM (COronary CT Angiography EvaluatioN For Clinical Outcomes: An InteRnational Multicenter registry) registry. Arterioscler Thromb Vasc Biol 2015;35:981-9. doi:10.1161/ATVBAHA. 114.304351

23 Windecker S, Kolh P, Alfonso F, et al. Authors/Task Force members. 2014 ESC/EACTS Guidelines on myocardial revascularization: The Task Force on Myocardial Revascularization of the European Society of Cardiology (ESC) and the European Association for Cardio-Thoracic Surgery (EACTS)Developed with the special contribution of the European Association of Percutaneous Cardiovascular Interventions (EAPCI). Eur Heart/2014;35:2541-619. doi:10.1093/eurheartj/ ehu278.

24 Dewey M, Zimmermann E, Deissenrieder F, et al. Noninvasive Coronary Angiography by 320-Row CT with Lower Radiation Exposure and Maintained Diagnostic Accuracy: Comparison of Results with Cardiac Catheterization in a Head-To-Head Pilot Investigation. Circulation 2009;120:867-75. Circulation 2009:120:867-75. doi:10.1161/CIRCULATIONAHA.109.859280.

25 Dewey M, Laule M, Krug L, et al. Multisegment and halfscan reconstruction of 16-slice computed tomography for detection of coronary artery stenoses. Invest Radiol 2004:39:223-9. doi:10.1097/01.rli.0000115201.27096.6e.

26 Leschka S, Kim CH, Baumueller S, et al. Scan length adjustment of CT coronary angiography using the calcium scoring scan: effect on radiation dose. A/R Am J Roentgenol 2010;194:W272-7. doi:10.2214/ AJR.09.2970

27 Zimmermann E, Dewey M. Whole-heart 320-row computed tomography: reduction of radiation dose via prior coronary calcium scanning. Rofo 2011;183:54-9. doi:10.1055/s-0029-1245629.

28 Gottlieb I, Miller JM, Arbab-Zadeh A, et al. The absence of coronary calcification does not exclude obstructive coronary artery disease or the need for revascularization in patients referred for conventional coronary angiography. J Am Coll Cardiol 2010;55:627-34. doi:10.1016/j.jacc.2009.07.072

29 Huda W, Ogden KM, Khorasani MR. Converting dose-length product to effective dose at CT. Radiology 2008;248:995-1003. doi:10.1148/ radiol.2483071964.

30 Leung KC, Martin Cl. Effective doses for coronary angiography. Br J Radiol 1996;69:426-31. doi:10.1259/0007-1285-69-821-426.

31 Ladenheim ML, Pollock BH, Rozanski A, et al. Extent and severity of myocardial hypoperfusion as predictors of prognosis in patients with suspected coronary artery disease. J Am Coll Cardiol 1986;7:464-71. doi:10.1016/S0735-1097(86)80454-5.

32 Schönenberger E, Schnapauff D, Teige F, Laule M, Hamm B, Dewey M. Patient acceptance of noninvasive and invasive coronary angiography. PLoS One 2007;2:e246. doi:10.1371/journal. pone.0000246.

33 Cox DR. Regression Models and Life-Tables. J R Stat Soc B 1972;34:187-220.

34 Napp A, Haase R, Laule M, et al. Computed Tomography versus Invasive Coronary Angiography: Design and Methods of the Pragmatic Randomised Multicentre DISCHARGE Trial. Eur Radiol 2016; in press.

35 Braunwald E. Unstable angina. A classification. Circulation 1989:80:410-4. doi:10.1161/01.CIR.80.2.410. 
36 Thygesen K, Alpert JS, Jaffe AS, et al. Joint ESC/ACCF/AHA/WHFTask Force for Universal Definition of Myocardial Infarction Authors/Task Force Members Chairpersons Biomarker Subcommittee ECG Subcommittee Imaging Subcommittee Classification Subcommittee Intervention Subcommittee Trials \& Registries Subcommittee Trials \& Registries Subcommittee Trials \& Registries Subcommittee Trials \& Registries Subcommittee ESC Committee for Practice Guidelines (CPG) Document Reviewers. Third universal definition of myocardial infarction. J Am Coll Cardiol 2012;60:1581-98. doi:10.1016/j.jacc.2012.08.001.

37 Hurley R. Can doctors reduce harmful medical overuse worldwide? BMJ 2014;349:g4289. doi:10.1136/bmj.g4289.

38 Lenzer J. Choosing Wisely: setbacks and progress. BMJ 2015;351:h6760. doi:10.1136/bmi.h6760.

39 Dewey M, Hamm B. Cost effectiveness of coronary angiography and calcium scoring using CT and stress MRI for diagnosis of coronary artery disease. Eur Radiol 2007:17:1301-9. doi:10.1007/s00330-006-0439-3.

40 Genders TS, Meijboom WB, Meijs MF, et al. CT coronary angiography in patients suspected of having coronary artery disease: decision making from various perspectives in the face of uncertainty. Radiology 2009;253:734-44. doi:10.1148/radiol.2533090507.

41 Hamilton-Craig C, Fifoot A, Hansen M, et al. Diagnostic performance and cost of CT angiography versus stress ECG--a randomized prospective study of suspected acute coronary syndrome chest pain in the emergency department (CT-COMPARE). Int J Cardiol 2014;177:867-73. doi:10.1016/j.ijcard.2014.10.090.

42 Winkens R, Dinant GJ. Evidence base of clinical diagnosis: Rational, cost effective use of investigations in clinical practice. BMJ 2002;324:783. doi:10.1136/bmj.324.7340.783.

43 Agus AM, McKavanagh P, Lusk L, et al. The cost-effectiveness of cardiac computed tomography for patients with stable chest pain. Heart 2016;102:356-62. doi:10.1136/heartinl-2015-308247.

44 Thom H, West NE, Hughes V, et al. CECaT study group. Costeffectiveness of initial stress cardiovascular MR, stress SPECT or stress echocardiography as a gate-keeper test, compared with upfront invasive coronary angiography in the investigation and management of patients with stable chest pain: mid-term outcomes from the CECaT randomised controlled trial. BMJ Open 2014;4:e003419. doi:10.1136/ bmjopen-2013-003419.

45 Geleijns J, Dewey M. Physics Background and Radiation Exposure. In: Dewey M, ed. Cardiac CT. Springer, 2014;58. doi:10.1007/978-3-642-41883-9 7

46 Sox HC Jr, , Margulies I, Sox CH. Psychologically mediated effects of diagnostic tests. Ann Intern Med 1981;95:680-5. doi:10.7326/0003-4819-95-6-680.

47 Halliburton SS, Abbara S, Chen MY, et al. SCCT guidelines on radiation dose and radiation dose-optimization strategies in cardiovascular CT. J Cardiovasc Comput Tomogr 2011;5:198-224.

48 Hoffmann U, Akers SR, Brown RK, et al. ACR Appropriateness Criteria Acute Nonspecific Chest Pain-Low Probability of Coronary Artery Disease. J Am Coll Radiol 2015;12(12 Pt A):1266-71. doi:10.1016/j. jacr.2015.09.004.
49 Taylor AJ, Cerqueira M, Hodgson JM, et al. American College of Cardiology Foundation Appropriate Use Criteria Task Force Society of Cardiovascular Computed Tomography American College of Radiology American Heart Association American Society of Echocardiography American Society of Nuclear Cardiology North American Society for Cardiovascular Imaging Society for Cardiovascular Angiography and Interventions Society for Cardiovascular Magnetic Resonance. ACCF/SCCT/ACR/AHA/ASE/ ASNC/NASCI/SCAI/SCMR 2010 appropriate use criteria for cardiac computed tomography. A report of the American College of Cardiology Foundation Appropriate Use Criteria Task Force, the Society of Cardiovascular Computed Tomography, the American College of Radiology, the American Heart Association, the American Society of Echocardiography, the American Society of Nuclear Cardiology, the North American Society for Cardiovascular Imaging, the Society for Cardiovascular Angiography and Interventions, and the Society for Cardiovascular Magnetic Resonance. J Am Coll Cardiol 2010;56:1864-94. doi:10.1016/j. jacc.2010.07.005.

50 Maurer MH, Zimmermann E, Schlattmann P, Germershausen C, Hamm $\mathrm{B}$, Dewey M. Indications, imaging technique, and reading of cardiac computed tomography: survey of clinical practice. Eur Radiol 2012;22:59-72. doi:10.1007/s00330-011-2239-7.

51 Chow BJ, Abraham A, Wells GA, et al. Diagnostic accuracy and impact of computed tomographic coronary angiography on utilization of invasive coronary angiography. Circ Cardiovasc Imaging 2009;2:1623. doi:10.1161/CIRCIMAGING.108.792572.

52 Pugliese F, Hunink MG, Gruszczynska K, et al. Learning curve for coronary CT angiography: what constitutes sufficient training? Radiology 2009;251:359-68. doi:10.1148/radiol.2512080384.

53 Chin S, Ong T, Chan W, et al. 64 row multi-detector computed tomography coronary image from a centre with early experience: first illustration of learning curve. J Geriatr Cardiol 2006;3:29-34

54 Andreini D, Pontone G, Pepi M, et al. Diagnostic accuracy of multidetector computed tomography coronary angiography in patients with dilated cardiomyopathy. I Am Coll Cardiol 2007:49:2044-50. doi:10.1016/j.jacc.2007.01.086.

55 Patel MR, Dai D, Hernandez AF, et al. Prevalence and predictors of nonobstructive coronary artery disease identified with coronary angiography in contemporary clinical practice. Am Heart J 2014;167:846-52. doi:10.1016/j.ahj.2014.03.001.

56 Patel N, Pal RS, Flores F, Budoff M. Utility of cardiac computed tomography angiography to exclude clinically significant obstructive coronary artery disease in patients after myocardial perfusion imaging. Am J Cardiol 2012;109:165-8. doi:10.1016/j. amjcard.2011.08.022.

Supplementary file: study protocol Supplementary file: additional analyses 